PHYSICAL REVIEW A 94, 039901(E) (2016)

\title{
Publisher's Note: Postquench dynamics and prethermalization in a resonant Bose gas [Phys. Rev. A 93, 033653 (2016)]
}

\author{
Xiao Yin and Leo Radzihovsky \\ (Received 6 September 2016; published 15 September 2016)
}

DOI: 10.1103/PhysRevA.94.039901

This paper was published online on 31 March 2016 with an incorrect reference. Reference [24] should read as "M. Srednicki, Phys. Rev. E 50, 888 (1994).” The reference has been corrected as of 7 September 2016. The reference is incorrect in the printed version of the journal. 